\title{
A DIRECT SYNTHESIS CONTROL STRATEGY FOR SINGLE-INPUT, SINGLE-OUTPUT NONLINEAR SYSTEM
}

\section{Ademola Stanford OLUFEMI}

This paper describes the strategy to design and modify a conventional controller by direct synthesis and the internal model control (IMC) methods for a single-input, single-output (SISO) process system. Both of these methods are the most widely used approaches, both in their simplicity and in their generality. The design of a direct synthesis controller based on a linear inversion of the non-linear process model about a steady-state was first considered. The particular linear design technique adopted resulted in a Proportional - Integral (PI) controller whose parameters are easily computed as a function of the parameters of the linear invertible model and the controller tuning parameter. The internal model control (IMC) methods were also considered to determine the fault from external disturbance. From this study, it was observed that under certain conditions both direct synthesis and internal model control methods are equal. Their use in the design of analog and digital controllers is presented by the method of the required model.

Key words: PID, direct-synthesis, IMC, required-medel, controller design.

\section{Introduction}

The automatic control of processes in order to ensure safe operation, reduce material and energy costs and meet required product specification is a commonly occurring problem in process industries. These processes are known to exhibit nonlinear dynamic behaviors. Thus, the linear, constant parameter controller that is usually designed from a linear approximation of the nonlinear process model cannot be expected to provide optimum performance over a wide range of system operating conditions. However, if a linear controller with a direct synthesis characteristics can be designed, then better system performance is possible with the linear, direct synthesis controller can be obtained.

Conventional PID Controllers according to [1-3] is used in over $95 \%$ of industrial process control systems. In most cases, these are PI type controllers. More than $80 \%$ of these control systems are poorly modified $[4,5]$. It is therefore clear that due attention should be given to the design of the relevant controllers and their adjustment.

The paper describes the development of a direct synthesis control strategy for single-input, single-output (SISO) nonlinear systems by two approaches that use general principles of dynamics inversion and which are often referred to in the literature as model-based approaches simple control system and is called a direct synthesis $[3,6]$. In these approaches, it is assumed that the regulated system model is known and that the control system model is presented respectively and appropriately chosen.

${ }^{1}$ Department of Chemical Engineering, Osmangazi University, Eskisehir, TURKEY (503520181006@ogrenci.ogu.edu.tr) https://orcid.org/0000-0003-4523-1454 
The first approach is based on a method and the second is based on a branched control circuit with an internal model and is called an internal model control, IMC method. In this study, only conventional controllers will be considered.

\section{General Regulatory Requirement}

A simple control system shown in Figure 1 is considered, where $G_{R}$ is a controller conversion, $G_{S} G_{D}$ control system control, $\mathrm{W}$ - a desired variable image, $\mathrm{Y}$ - a controlled variable image, $\mathrm{V}$ - a variable error image. As an independent variable, it is necessary to consider complex variables $\mathrm{s}$ for continuous control system and for a discrete complex variable $\mathrm{z}$.

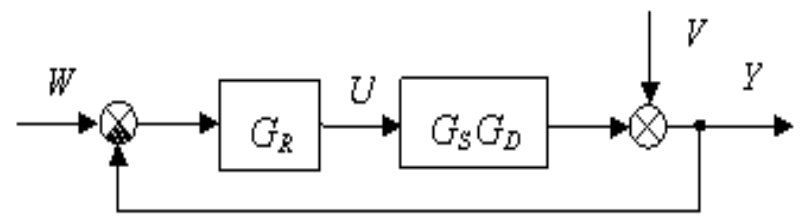

Figure 1: Simple control system

It is assumed that the regulated system is stable and that it consists of an invertible $G_{S}$ transfer portion and a $G_{D}$ noninvertible portion that is most often represented by a transport delay $\left(\left|G_{D}\right|=1\right)$. The goal of regulation can be expressed by a simple relationship:

$$
Y \rightarrow W
$$

From which the controller has a dual task of ensuring that the desired variable $W$ is monitored by the controlled variable $Y$ while suppressing the negative effect of the failure $V$ on the control system. Of course, the control system must be stable and the control target (1) must be filled with the specified quality.

For the control system in Figure 1.

$$
\begin{aligned}
Y & =G_{w y}^{*} W+G_{v y}^{*} V \\
G_{w y}^{*} & =\frac{G_{R} G_{S} G_{D}}{1+G_{R} G_{S} G_{D}} \\
G_{v y}^{*} & =\frac{1}{1+G_{R} G_{S} G_{D}}=1-G_{w y}^{*}
\end{aligned}
$$

Where the control transmission is, - the fault transmission. An asterisk $(*)$ will further indicate the values required, recommended, and/or optimal, $G_{v y}^{*} G_{w y}^{*}$.

Given the existence of the non-invertible part $G_{D}$, the objective of regulation (1) will be fulfilled if it applies [7].

$$
G_{w y}^{*} \rightarrow G_{D}, \quad G_{v y}^{*} \rightarrow 1-G_{D}
$$

In the absence of a non-invertible part, i.e., $G_{D}=1$, conditions (5) are simplified,

$$
G_{w y}^{*} \rightarrow 1, \quad G_{v y}^{*} \rightarrow 0
$$

Since (4) is true, it is clear that if in relations (5) and (2) respectively. (6) the condition for transmission of the control is fulfilled, then the corresponding condition for the transmission of the fault will automatically be fulfilled. Therefore, only control transmission will be considered further, 
$G_{w y}^{*} G_{v y}^{*} G_{w y}^{*}$.

\subsection{Direct Synthesis Method}

Based on the relation (3), the transfer of the controller can be determined

$$
G_{R}=\frac{1}{G_{S} G_{D}} \frac{G_{w y}^{*}}{1-G_{w y}^{*}}
$$

Which will ensure the required control transfer. $G_{w y}^{*}$. Equation (7) is called synthesis equation and serves to design regulators by direct synthesis.

Since the non-invertible portions $G_{D}$ cannot be disposed of, the required control transmission is assumed to be in the shape.

$$
G_{w y}^{*}=G_{w y} G_{D}
$$

Where $G_{w y}$ is the invertible portion of the required control transmission most often in the form of a proportional element with first or second order inertia.

It is the part of the $G_{w y}$ that the required steering transmission (8) differs from the "ideal" steering transmission.

$$
G_{w y}^{*}=G_{D}
$$

From equation (5), which is responsible for equation (6) for $G_{D}=1$. Putting equation (8) into equation (7)

$$
G_{R}=\frac{G_{w y}}{G_{S}-G_{S} G_{w y} G_{D}}
$$

From equation (10), it requires that the transfer function of the controller be selected and the transmission portion is an invertible $G_{S}$ un-invertible part $G_{D}$ regulated system is suitably adjusted so that the resulting transmission controller $G_{R}$ was physically realizable and conventional (Table 1) $G_{w y}^{*}$ . These are the drawbacks of direct synthesis, but on the other hand, it is a visual and very simple method.

\section{Example 1:}

By direct synthesis, it is desirable to design a suitable conventional regulator for the transmission system.

$$
G_{S}(s)=\frac{k_{1}}{T_{1} s+1}
$$

\section{Solution:}

It follows from the transfer of the control system (11) that $G_{D}(s)=1$ and therefore for the selected control function desired.

$$
G_{w y}^{*}=G_{w y}=\frac{1}{T_{w} s+1}
$$

A conventional analogue PI controller is obtained after it is set to equation (10)

$$
G_{R}(s)=\frac{T_{1} s+1}{k_{1} T_{w} s}=k_{p}^{*}\left(1+\frac{1}{T_{1}^{*} s}\right)
$$


Where $k_{p}^{*}=\frac{T_{1}^{*} s}{k_{1} T_{w}}, T_{1}^{*}=T_{1}$

The time constant, $T_{w}$ of the control system serves as a "tuning" parameter, which can be used to fine-tune the control process, for example, the limitation of the control variable, the control time, etc. Figure 2, shows the responses of the controlled variable $y(t)$ to the unit step changes of the desired value $w(t)$ for $k_{1}=1, T_{1}=5$ and $T_{w}=1,2$ and $3 \mathrm{sec}$.

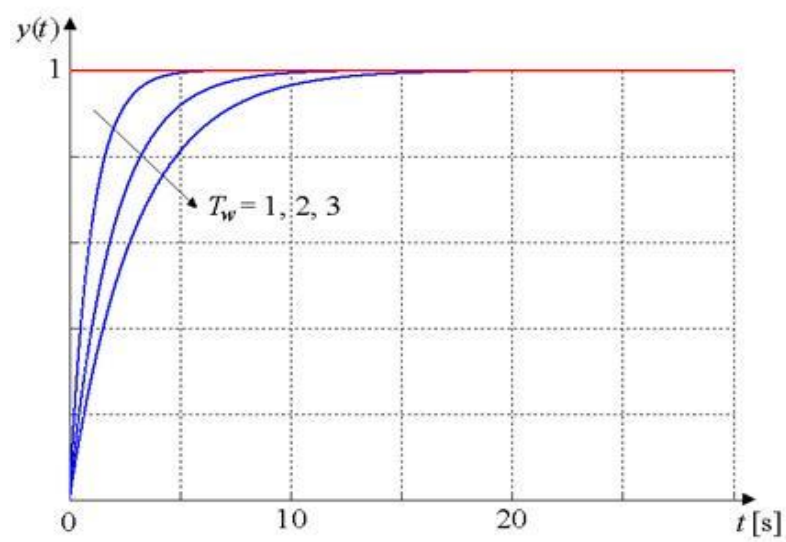

Figure 2: Transition characteristics of control system - example 1

\section{Example 2}

The controlled system has a transfer

$$
\frac{k_{1}}{T_{1} s+1} e^{-T_{d} s}
$$

A suitable controller should be designed by direct synthesis.

\section{Solution:}

It follows from equation (14) that the regulated system has both an invertible and a non-invertible part.

$$
G_{S}(s)=\frac{k_{1}}{T_{1} s+1}
$$

Where $G_{D}(s)=e^{-T_{d} s}$

For the required steering control in shape

$$
G_{w y}^{*}=\frac{1}{T_{w} s+1} e^{-T_{d} s}, \quad G_{w y}(s)=\frac{1}{T_{w} s+1}, \quad G_{D}(s)=e^{-T_{d} s}
$$

Substituting equations (15) and (16) into equation (10),

$$
G_{R}(s)=\frac{T_{1} s+1}{k_{1}\left(T_{1} s+1-e^{-T_{d} s}\right)}
$$

Using approximation,

$$
e^{-x} \approx 1-x
$$

The transfer function in equation (17) can be adapted to a shape corresponding to a conventional 
PI controller.

$$
\begin{gathered}
G_{R}(s)=\frac{T_{1} s+1}{k_{1}\left(T_{w}+T_{d}\right) s}=k_{p}^{*}\left(1+\frac{1}{T_{1}^{*} s}\right) \\
k_{p}^{*}=\frac{T_{1}^{*}}{k_{1}\left(T_{w}+T_{d}\right)}
\end{gathered}
$$

Where $T_{1}^{*}=T_{1}$.

Also here, the time constant $T_{w}$ has the meaning of a "tuning" parameter, which in this case affects not only the speed and overshoot of the response, but also the robustness of the entire control system, and $T_{w}=T_{d}$ is the default value.

The responses of the controlled variable $y(t)$ to the unit step changes of the position of the desired variable $w(t)$ for $k_{1}=1, T_{1}=T_{d}=5$ and $T_{w}=3,5$ and 7 seconds as presented in Figure $3 \mathrm{a} \&$ b.
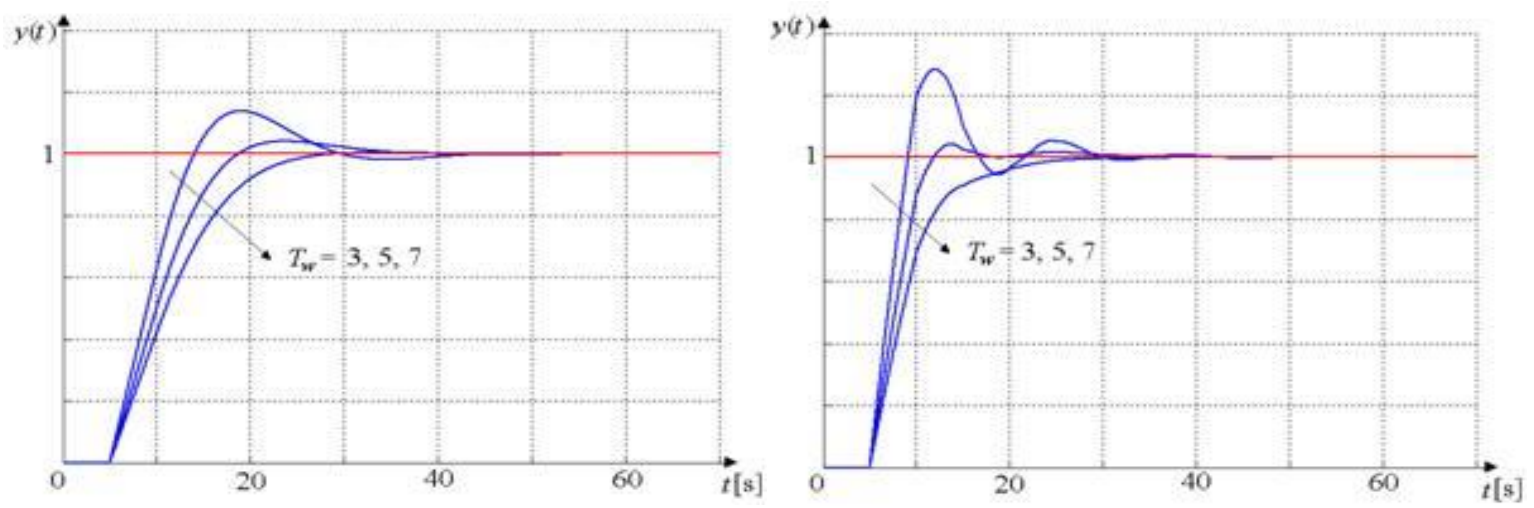

Figure 3: Transition characteristics of control system from example 2: a) for PI controller, b) for PID controller

If the form of transmission of the controlled system in equation (14) is used,

$$
\begin{gathered}
\frac{k_{1}}{T_{1} s+1} e^{-T_{d} s} \approx \frac{k_{1}}{\left(T_{1} s+1\right)\left(\frac{T_{d}}{2} s+1\right)} e^{-\frac{T_{d}}{2} s} \\
G_{S}(s)=\frac{k_{1}}{\left(T_{1} s+1\right)\left(\frac{T_{d}}{2} s+1\right)}, \quad G_{D}(s)=e^{-\frac{T_{d}}{2} s}
\end{gathered}
$$

Then, after substituting equations (16) and (21) into equation (10), it gets

$$
G_{R}(s)=\frac{\left(T_{1} s+1\right)\left(\frac{T_{d}}{2} s+1\right)}{k_{1}} \frac{1}{T_{w} s+1-e^{-\frac{T_{d}}{2} s}}
$$

By using an approximation (18), a transmission corresponding to the PID controller is obtained

$$
\begin{gathered}
G_{R}(s)=\frac{\left(T_{1} s+1\right)\left(\frac{T_{d}}{2} s+1\right)}{k_{1}\left(T_{w}+\frac{T_{d}}{2}\right) s}=k_{p}^{*}\left(1+\frac{1}{T_{I}^{*} s}+T_{D}^{*} s\right) \\
k_{p}^{*}=\frac{T_{I}^{*}}{k_{1}\left(T_{w}+\frac{T_{d}}{2}\right)}
\end{gathered}
$$


Where $T_{I}^{*}=T_{1}+\frac{T_{d}}{2}$ and $T_{D}^{*}=\frac{T_{1} \frac{T_{d}}{2}}{T_{1}+\frac{T_{d}}{2}}$

The responses of the controlled variable $y(t)$ to the unit step changes of the position of the desired variable $w(t)$ for $k_{1}=1, T_{1}=T_{d}=5$ and $T_{w}=3,5$ and 7 seconds as shown in Figure $3 \mathrm{~b}$.

It can be seen from the comparison of the control variable $y(t)$ in Figure 3 that the PID controller gives a faster response than the PI controller.

\subsection{Internal Model Control (IMC) Method}

Figure 4 is a branched control system with internal model control where the transfer controller controls the system with an internal model control (short - transmission controller IMC), $G_{F}$ - control filter; $G_{S M}$ - control invertible portion $G_{S}, G_{D M}$ - transfer model non-invertible part $G_{D} . G_{R}^{I M C}$.

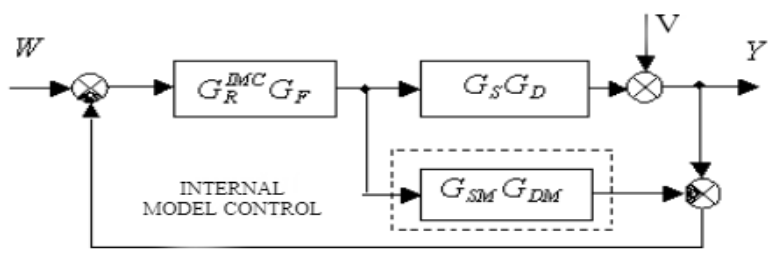

Figure 4: Control system with internal model control

If the internal model is ideally identical to the regulated system, i.e.

$$
G_{S M}=G_{S}, \text { then } G_{D M}=G_{D}
$$

Then the internal model control system can be transformed into the scheme of Figure 5, from which it is clear that the branching serves primarily to identify $\mathrm{V}$ fault. Recall from equation (2), we have:

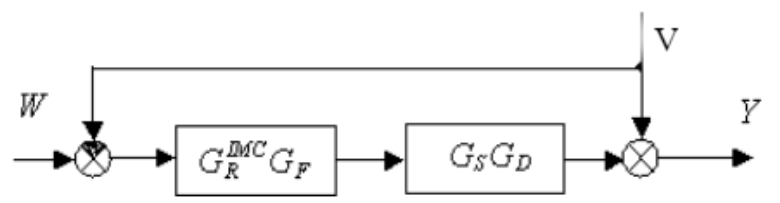

Figure 5: Transformed control system with internal model at equation (24)

$$
\begin{gathered}
Y=G_{w y}^{*} W+G_{v y}^{*} V \\
G_{w y}^{*}=G_{R}^{I M C} G_{F} G_{S} G_{D} \\
G_{v y}^{*}=1-G_{R}^{I M C} G_{F} G_{S} G_{D}=1-G_{w y}^{*}
\end{gathered}
$$

Also in this case, only the control transfer will be considered in view of validity (27).

In order to meet the objective of regulation (1), equations (5) and (3) must apply (6) for $G_{D}=1$. Obviously, these conditions will be met for:

$$
G_{R}^{I M C} \rightarrow \frac{1}{G_{S}} \text { and } G_{F} \rightarrow 1
$$

Because part of $G_{S}$ is stable and invertible, theoretically its inversion can be done, but that does not mean that it can be physically implemented. A well-chosen filter allows the physical implementation of a transmission member: 


$$
G_{R}^{I M C} G_{F}=\frac{G_{F}}{G_{S}}
$$

After substituting (29) into (26), the resulting control of the control system with the internal model is obtained as:

$$
G_{w y}^{*}=G_{F} G_{D}
$$

A comparison of the relations (30) and (8) shows that the filter $G_{F}$ performs a similar function in the internal model control system (Figure 4) as part of the $G_{w y}$ control transmission in a simple control system (Figure 1).

In order to design a conventional controller by the internal model control method, the branched internal model control system of Figure 4 must be transformed into the equivalent simple control system of Figure 1.

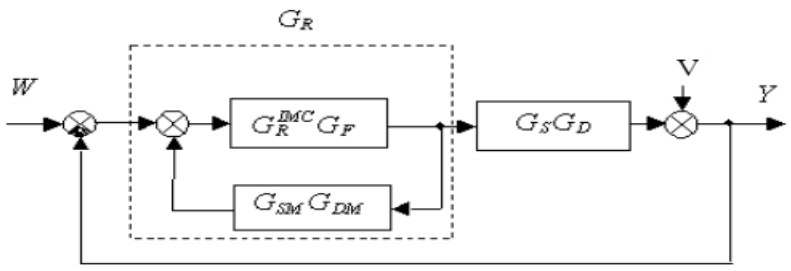

Figure 6: Control system with internal model transformed into a simple control system

Based on Figure 6, it is already possible to determine the corresponding transmission of the controller in a simple control system.

$$
G_{R}=\frac{G_{R}^{I M C} G_{F}}{1-G_{R}^{I M C} G_{F} G_{S M} G_{D M}}
$$

If the internal model is ideally matched to the controlled system, i.e. when the conditions (24) and validity (29) are met, the relationship for the controller transmission in the simple control system is obtained.

$$
G_{R}=\frac{1}{G_{S}} \frac{G_{F}}{1-G_{F} G_{D}}
$$

A comparison of the relationships (32) and (10) shows that for:

$$
G_{F}=G_{w y}
$$

The internal model method and the direct synthesis method lead to the same controller in a simple control system. That is, when designing conventional controllers, both methods are equivalent and indistinguishable essentially when the condition of equation (33) is met. Therefore, for the internal model method, the same constraints apply to the conventional controller design as for the direct synthesis method.

In fact, both methods are considerably more general than is stated in this article, limiting it to conventional controllers [7-11].

Both the direct synthesis method and the internal model control method can also be used for unstable controlled systems $[8,12]$. The internal model control method makes it easier to ensure the robustness of the control system $[9,13]$.

\subsection{Method of Desired Model}

The method of the required model (formerly called dynamics inversion method) is the original method, which was designed, developed by [14-16]. It is a method using direct synthesis. At present, the required model method is extended to a larger number of different regulated systems [17]. 


\subsubsection{Analog control system design}

It is assumed that the required control system control with analog controller (hence the name of the method) has the form of an isochronous model:

$$
G_{w y}(s)=\frac{a}{s+a e^{-T_{d} s}} e^{-T_{d} s}
$$

i.e.

$$
G_{w y}(s)=\frac{a}{s+a e^{-T_{d} s}}, \quad G_{D}(s)=e^{-T_{d} s}
$$

Where $a$ is the gain of the open control loop.

The chosen shape of the desired steering transmission (34) is the simplest shape that allows the choice of amplification of the open control system and to achieve different response patterns from aperiodic to oscillating, see Figure 7.

Unlike conventional direct synthesis methods and the internal model, the required control transmission (34) has a shape that directly leads to conventional controller transmissions for appropriate transfers of regulated systems. There need not output approximation to time delay in the transmission controller and therefore there is also a greater accuracy for the different values of the gain control system of the open.

Amplification of the open control system and ensuring the required relative overshoot can be approximately analytically determined assuming the dominance of a pair of characteristic quasi-multipoints of the required steering transmission (34).

$$
N(s)=s+a e^{-T_{d} s}
$$

And comparing it with the corresponding pair of poles of the proportional oscillating member of the second order or by digital simulation. The difference between analytical and simulation solutions for relative overshoot from 0 to 0.5 (0 to $50 \%$ ) does not exceed $2 \%$.

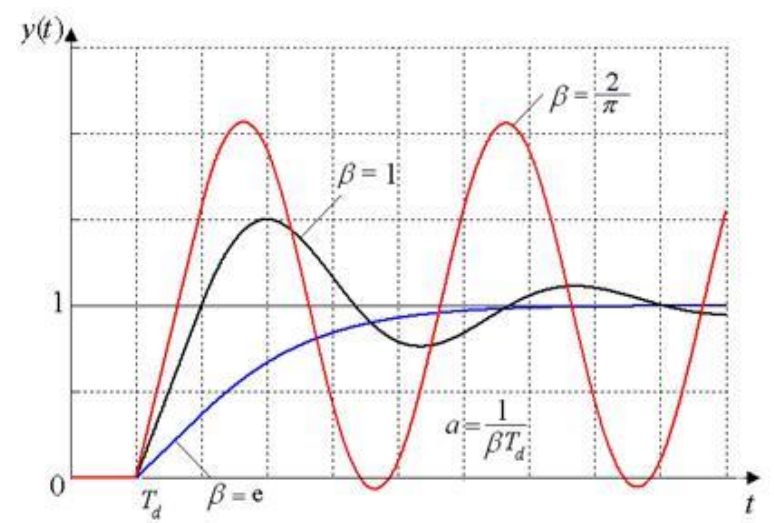

Figure 7: Transition characteristics of the control system with the required control transmission (34) for different

$$
\text { values of } a(\beta) \text {. }
$$

Values of the coefficient $\beta$ for the calculation of the open-loop control gain and the relation.

$$
a=\frac{1}{\beta T_{d}}
$$

For the required relative overshoot detailed simulations are given in Table 2.

The procedure for designing and adjusting conventional analogue controllers using the required model is shown in Example 3. All relationships in Table 3 (for $T=0$ ).

\section{Example 3}

For a regulated transmission system 


$$
\frac{k_{1}}{\left(T_{1} s+1\right)\left(T_{2} s+1\right)} e^{-T_{d} s}, \quad T_{1}>T_{2}
$$

It is necessary to design a suitable conventional analogue regulator and to adjust it so that when the position of the desired variable is stepped $w(t)$ approximately the aperiodic control process is reached.

\section{Solution:}

It is clear from the transfer function of the control system (38) that:

$$
G_{S}(s) \frac{k_{1}}{\left(T_{1} s+1\right)\left(T_{2} s+1\right)}, \quad G_{D}(s)=e^{-T_{d} s}
$$

After substituting (39) and (35) to (10) and adjusting, the PID controller is transmitted.

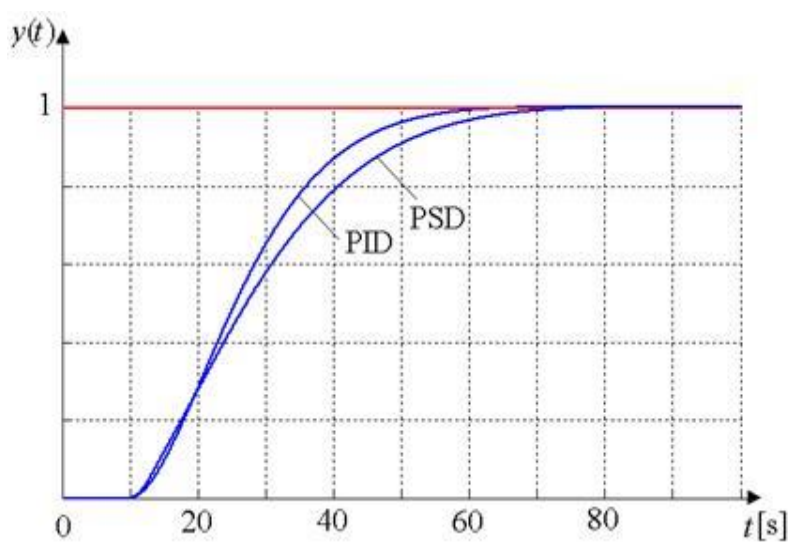

Figure 8: Transition characteristics of control system with analog PID and digital PSD controller adjusted by the

$$
\text { required model for } a^{*}=0 \text {. Examples } 3 \text { and } 4
$$

\subsubsection{Digital control system design}

It is believed that the desired control system is controlled in shape with the digital controller.

$$
G_{w y}^{*}(z)=\frac{a T}{z-1+a T z^{-d}} z^{-d}
$$

i.e.

$$
G_{w y}(z)=\frac{a T}{z-1+a T z^{-d}}, \quad G D(z)=z^{-d}
$$

Where $d$ is a discrete relative transport delay (an integer is assumed for simplicity), $T$ is a sampling period.

Furthermore, it is also assumed that the quantization error is negligibly small, and therefore discrete and digital terms are considered equivalent.

The shape of the expected control system which is controlled by the digital controller (44) corresponds to the desired control system transmitted with the analog controller (34) and therefore the choice of the gain of the open control system and similar waveforms as in Figure 7 can also be achieved.

Unlike a control system with an analogue gain controller, an open control loop and providing some overshoot, it is not possible to determine analytical, but only simulation, and it can be expressed by:

$$
a=\frac{1}{\alpha T+\beta T_{d}}
$$

Where $\alpha$ is the coefficient determined by the digital simulation for the desired relative overshoot, see Table 2.

Discretized Transmission of a Regulated System $G_{S}(z) G_{D}(z)$ can be determined based on the relationship using a zero order sampler and shaper. 


$$
G_{S}(z) G_{D}(z)=\frac{z-1}{z} Z\left\{\begin{array}{l}
L^{-1}\left\{\frac{1}{s} G_{S}(s) G_{D}(s)\right\} \\
\left.\right|_{t=k T}
\end{array}\right\}
$$

Where $k$ is the relative discrete time, $\mathrm{Z}$ - the Z-transform operator, $\mathrm{L}$ - the L-transform operator.

If the non-invertible part of the regulated system $G_{D}(s)$ represents only the traffic delay, i.e.

$$
G_{D}(s)=e^{-T_{p} s} \Rightarrow G_{D}(z)=z^{-d}
$$

Then the discretized transmission of the regulated system can be determined from a simpler relationship,

$$
G_{S}(z) G_{D}(z)=\frac{z-1}{z} Z\left\{\left.L^{-1}\left\{\frac{1}{s} G_{S}(s)\right\}\right|_{t=k T}\right\} z^{-d}
$$

The procedure for designing and adjusting conventional digital controllers using the required model is shown in Example 4. All relationships in Table 3 (for $\mathrm{T}>0$ ).

\section{Example 4}

For the controlled system (38) of Example 3, it is desirable to design a suitable conventional digital controller and adjust it so that, when the position of the desired variable is stepped, an approximately limit a periodic control process is provided.

\section{Solution:}

Using the relationships in equations (38) and (49), a discretized transmission of the regulated system is obtained

$$
\begin{gathered}
G_{S}(z) G_{D}(z)=\frac{k_{1}(A z+B)}{\left(z-c_{1}\right)\left(z-c_{2}\right)} z^{-d} \\
c_{1}=e^{-\frac{\Gamma}{\Gamma_{1}}}, \quad c_{2}=e^{-\frac{\Gamma}{\Gamma_{2}}} \\
A=\frac{T_{1} c_{1}-T_{2} c_{2}}{T_{2}-T_{1}}+1, \quad B=c_{1} c_{2}+\frac{T_{1} c_{2}-T_{2} c_{1}}{T_{2}-T_{1}}
\end{gathered}
$$

Substituting the relationships (45) and (50) into (10) and adjusting the transmission,

$$
G_{R}(z)=\frac{\alpha T\left(c_{1}+c_{2}-2 c_{1} c_{2}\right) z}{k_{1}(A z+B)} *\left(1+\frac{1-c_{1}-c_{2}+c_{1} c_{2}}{c_{1}+c_{2}-2 c_{1} c_{2}} \frac{z}{z-1}+\frac{c_{1} c_{2}}{c_{1}+c_{2}-2 c_{1} c_{2}} \frac{z-1}{z}\right)
$$

Which is similar to the transmission of a conventional PSD (see Table 1) with adjustable parameters.

$$
\begin{gathered}
k_{p}^{*}(z)=\frac{\alpha T\left(c_{1}+c_{2}-2 c_{1} c_{2}\right)}{k_{1}(A z+B)} \\
T_{1}^{*}=\frac{c_{1}+c_{2}-2 c_{1} c_{2}}{1-c_{1}-c_{2}+c_{1} c_{2}} T \\
T_{D}^{*}=\frac{c_{1} c_{2}}{c_{1}+c_{2}-2 c_{1} c_{2}} T
\end{gathered}
$$

Digital controller gain is a function of the complex variable $\mathrm{z}$ and, in addition, at the small sampling period $T$, the so-called "ringing" problem arises because $k_{p}^{*}(z)$.

$$
T \rightarrow 0 \Rightarrow z_{1}=-\frac{B}{A} \rightarrow-1
$$

The bell ring of $z_{1}$ causes an impermissible oscillation of the actuating variable. Both of these problems can be eliminated by the representation of the complex variable-dependent gain from constant 
gain $k_{p}^{*}(z)$.

$$
k_{p}^{*}=\lim _{T \rightarrow 0} k_{p}^{*}(z)=\frac{\alpha^{*} T_{1}^{*}}{k_{1}}
$$

For $T>0$, relations (53) and (56) converge to the corresponding relationships for the analog PID controller (41).

Relationships (53) are not very suitable for practical use. Using approximation:

$$
e^{-\chi}=\frac{1-\frac{\chi}{2}}{1+\frac{\chi}{2}}
$$

They can be easily simplified, they are given (see Table 3, line 4):

$$
\begin{aligned}
& T_{1}^{*} \approx T_{1}+T_{2}-T \\
& T_{D}^{*} \approx \frac{T_{1} T_{2}}{T_{1}+T_{2}}-\frac{T}{4}
\end{aligned}
$$

It remains to be determined the gain of the open control loop and $T_{D}^{*}$, which will provide approximately the limit a periodic control process. It can be determined by assuming the dominance of a stable double real pole. The gains of $\alpha T_{1}^{*}$ pole are obtained by solving a system of equations, $z_{1}^{*} z_{2}^{*}$.

$$
N(z)=0, \quad \frac{d N(z)}{d z}=0
$$

Where:

$$
N(z)=z^{d+1}-z^{-d}+\alpha T
$$

It is a characteristic polynomial regulating system with a digital controller for the expected model method [it is the denominator of the desired control transmission (44) multiplied by $z^{d}$ ].

The solution (59) for (60) is obtained:

$$
\begin{aligned}
& z_{2}^{*}=\frac{d}{d+1} \\
& \alpha^{*}=\frac{1}{T} \frac{1}{d+1}\left(\frac{d}{d+1}\right)^{d}
\end{aligned}
$$

For the amplification of the open control system (62) it is advantageous to use an approximation.

$$
\alpha^{*}=\frac{1}{T} \frac{1}{d+1}\left(\frac{d}{d+1}\right)^{d} \approx \frac{1}{(4-e) T+e T_{d}}
$$

Whose inaccuracy for $d=0.5$ is better than $2 \%$ [see equation (46)].

From formula (63), it can be seen that for $T>0$, the gain of the open control loop and $\alpha^{*}$ with the analog PID controller providing the cut-off a periodic control step, see (43).

All results also apply to $T_{1}=T_{2}$.

The response to step change of the reference value for $k_{1}=1, T=3, T_{1}=T_{d}=10$ and $T_{2}=5$ seconds is shown in Figure 8. The response has been slowed down by the PSD.

\subsubsection{Modification of Control Systems Transmissions}

The desired model method is for a relative overshoot of less than $20 \%$ robust and meets all the more important criteria recommended for the design and adjustment of regulators. The approach using empirical "half rule" is very simple, which will be presented in a simplified form.

Assuming the transmission of the regulated system has a shape with unstable zeros: 


$$
G_{S}(s) G_{D}(s)=\frac{\prod_{j}\left(1-\tau_{j 0} s\right)}{\prod_{i}\left(T_{i 0} s-1\right)} e^{-\Gamma_{2 D} s}
$$

Where $T_{i 0} \geq T_{i+1,0} \geq 0, \quad \tau_{j 0} \geq 0$

Then on the basis of the "half rule" for replacement transmission

$$
G_{S}(s) G_{D}(s)=\frac{1}{T_{1} s+1} e^{-\Gamma_{2 D} s}
$$

Which can be transformed to:

$$
T_{1}=T_{10}+\frac{T_{20}}{2}, \quad T_{d}=T_{d 0}+\frac{T_{20}}{2}+\sum_{i 23} T_{i 0}+\sum_{j} \tau_{j 0}
$$

Respectively for transmission:

$$
\begin{gathered}
G_{S}(s) G_{D}(s)=\frac{1}{\left(T_{1} s+1\right)\left(T_{2} s+1\right)} e^{-\Gamma_{2} s} \\
T_{1}=T_{10}, \quad T_{2}=T_{20}+\frac{T_{30}}{2}, \quad T_{d}=T_{d 0}+\frac{T_{30}}{2}+\sum_{i 24} T_{i 0}+\sum_{j} \tau_{j 0}
\end{gathered}
$$

\section{Example 5}

By using the required model method, the PI and PID analogue controllers for the transmission system must be adjusted.

$$
G_{S}(s)=\frac{2(1-2 s)}{(10 s+1)(5 s+1)(4 s+1)(s+1)}
$$

So that the relative overshoot is about $5 \%$. All time constants are in seconds.

\section{Solution:}

For a regulated system (68): $G_{D}(s)=1, k_{1}=2, T_{10}=10, T_{20}=5, T_{30}=4, T_{40}=1$.

Based on Table 2 for $d=0.05$ which determines the coefficient $\beta=1,944$.

\section{Controller Application}

\subsection{PI controller}

In accordance with Table 3 (line 2) the transmission of the control system is to take the form (66a), and therefore, on the basis of (66b) can be written as:

$$
\begin{aligned}
& T_{1}=T_{10}+\frac{T_{20}}{2}=10+\frac{5}{2}=12.5 \mathrm{~s} \\
& T_{d}=T_{d 0}+\frac{T_{20}}{2}+T_{30}+T_{40}+\tau_{10}=0+\frac{5}{2}+4+1+2=9.5 \mathrm{~s} \\
& T_{1}^{*}=T_{1}=12.5 \mathrm{~s} \\
& k_{p}^{*}=\frac{T_{1}^{*}}{\beta T_{d} k_{1}}=\frac{12.5}{1,944 \times 9.5 \times 2}=0.34
\end{aligned}
$$

\subsection{PID controller}

Recall from Table 3 (line 4), the transmission of the control system is to take the form (66a), and therefore, based on (66b), can be written:

$$
T_{1}=T_{10}=10 s, T_{2}=T_{20}+\frac{T_{30}}{2}=5+\frac{4}{2}=5 s
$$




$$
\begin{aligned}
& T_{d}=T_{d 0}+\frac{T_{30}}{2}+T_{40}+\tau_{10}=0+\frac{4}{2}+1+2=5 s \\
& T_{1}^{*}=T_{1}+T_{2}=10+7=17 \mathrm{~s}, T_{D}^{*}=\frac{T_{1} T_{2}}{T_{1}+T_{2}}= \\
& \frac{10 \times 7}{10+7}=4.12 \mathrm{~s} \\
& k_{p}^{*}=\frac{T_{1}^{*}}{\beta T_{d} k_{1}}=\frac{17}{1,944 \times 5 \times 2}=0.87
\end{aligned}
$$

The responses to the step change of the desired magnitude are shown in Figure 9. Given the very rough adjustment of the default transmission of the control system, this is a very good result.

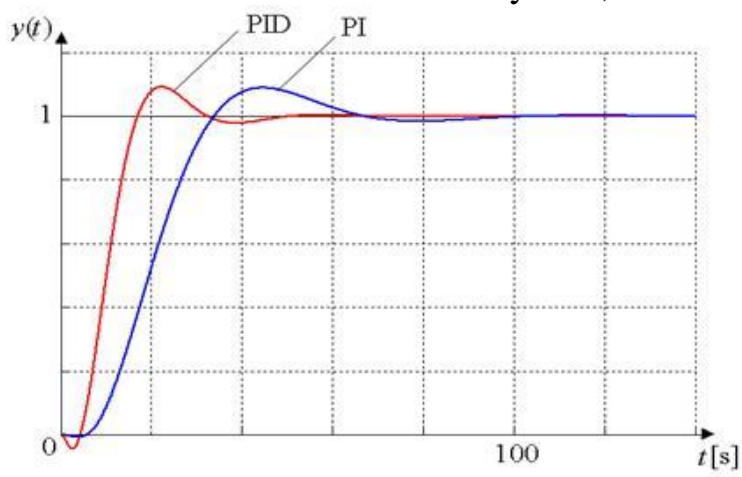

Figure 9: Transition characteristics of the control system with analog PI and PID controllers - example 5

\subsection{Procedure For Modifying The Controllers Using The Required Model}

a) Modify the transmission of a regulated system to one of the basic forms listed in Table 3.

b) In the case of an analog controller, consider $T=0$ in all relations and, in the case of a digital controller, select a suitable sampling period $T>0$.

c) For the desired relative overshoot based on Table 2 to determine the coefficients $a$ and $b$ and then for the recommended controller in accordance with Table 3 , calculate the values of its adjustable parameters.

d) In terms of the required relative overshoot, the method of the desired model in the case of analog controllers is quite accurate. In the case of digital controllers, its inaccuracy is on average about $5 \%$. This is due to the simplification of exponential function and complex relationships, see example 4. If the initial transmission of the regulated system is adjusted, then the accuracy is of course smaller.

\section{Conclusion}

Two approaches to designing and adjusting conventional controllers, namely the direct synthesis method and the internal model method, are briefly described and explained in the paper. It is shown that, with certain assumptions, both approaches are equivalent.

The method of the required model, which is described in more detail illustrates the use of the direct synthesis method for the design and adjustment of both analog and digital conventional controllers.

\section{References}

[1] Åström, K.J., Hägglund, T., PID controllers: theory, design, and tuning. ISA-The Instrumentation, Systems, and Automation Society Research Triangle Park, NC, 1995.

[2] Åström, K.J., Hägglund, T., Astrom, K.J., Advanced PID control. ISA-The Instrumentation, Systems, and Automation Society Research Triangle Park, NC, 2006. 
[3] Olufemi, A.S., Ogbeide, S.E. (2017). Dynamics and Control System Design for Biodiesel Transesterification Reactor. International Robotics \& Automation Journal, 2(6), 1-6.

[4] O'Dwyer, A., Handbook of PI and PID controller tuning rules. Imperial College Press, 2009.

[5] do Val, J.B., Souto, R.F., (2017). Modeling and control of stochastic systems with poorly known dynamics. IEEE Transactions on Automatic Control, 62(9), 4467-4482.

[6] Ogunnaike, B., Ray, W., Process Modeling, Dynamics and Control. ed: Oxford Univ Press, New York, USA, 1994.

[7] Chen, D., Seborg, D.E., (2002). PI/PID controller design based on direct synthesis and disturbance rejection. Industrial \& engineering chemistry research. 41(19), 4807-4822.

[8] Jung, C.S., Song, H.K., Hyun, J.C., (1999b). A new direct-synthesis tuning method for picontrollers. The Canadian Journal of Chemical Engineering, 77(1), 180-185.

[9] Rivera, D.E., Morari, M., Skogestad, S., (1986). Internal model control: PID controller design," Industrial Engineering Chemistry Process Design Development, 25(1), 252-265, 1986.

[10] Wang, Q.G., Hang, C.C., Yang, X.P., (2001). Single-loop controller design via IMC principles. Automatica, 37(12), 2041-2048.

[11] Wang, Y.C., Sheu, D., Lin, C.E., (2015). A unified approach to nonlinear dynamic inversion control with parameter determination by eigenvalue assignment. Mathematical Problems in Engineering, 2015.

[12] Tan, W., Marquez, H.J., Chen, T., (2003). IMC design for unstable processes with time delays. Journal of Process Control, 13(3), 203-213.

[13] Rivera, D.E., Internal model control: a comprehensive view," A. S. University, Ed., ed. Arizona, 19, 1999.

[14] Rao, A.S., Rao, V., Chidambaram, M., (2009). Direct synthesis-based controller design for integrating processes with time delay. Journal of the Franklin Institute, 346(1), 38-56.

[15] Adams, R.J., Banda, S.S., (1993). Robust flight control design using dynamic inversion and structured singular value synthesis. IEEE Transactions on Control Systems Technology, 1(2), 80-92.

[16] Dighe, Y.N., Kadu, C.B., Parvat, B.J., (2014). Direct Synthesis Approach for Design of PID Controller. International Journal of Application or Innovation in Engineering \& Management, 3(5), 161-167.

[17] Ajmeri, M., Ali, A., (2015). Direct synthesis based tuning of the parallel control structure for integrating processes. International Journal of Systems Science, 46(13), 2461-2473.

\section{APPENDIX}

Table 1: Conventional controller transmissions

\begin{tabular}{ccc}
\hline Controller Type & Analog controller & Digital controller \\
\hline P & $k_{p}$ & $k_{p}$ \\
\hline PI & $k_{p}\left(1+\frac{1}{T_{I} s}\right)$ & $k_{p}\left(1+\frac{T z}{T_{I} z-1}\right)$ \\
\hline PD & $k_{p}\left(1+T_{D} s\right)$ & $k_{p}\left(1+\frac{T_{D} z-1}{T z}\right)$ \\
\hline
\end{tabular}


PID

$$
k_{p}\left(1+\frac{1}{T_{I} s}+T_{D} s\right) \quad k_{p}\left(1+\frac{T z}{T_{I} z-1}+\frac{T_{D} z-1}{T z}\right)
$$

Table 2: Coefficients of $a$ and $b$ for the required comparative overshoot

\begin{tabular}{c|ccccccccccc}
\hline Time & $\mathbf{0}$ & $\mathbf{0 . 0 5}$ & $\mathbf{0 . 1 0}$ & $\mathbf{0 . 1 5}$ & $\mathbf{0 . 2 0}$ & $\mathbf{0 . 2 5}$ & $\mathbf{0 . 3 0}$ & $\mathbf{0 . 3 5}$ & $\mathbf{0 . 4 0}$ & $\mathbf{0 . 4 5}$ & $\mathbf{0 . 5 0}$ \\
\hline$a$ & 1,282 & 40.984 & 0.884 & 0.832 & 0.763 & 0.697 & 0.669 & 0.640 & 0.618 & 0.599 & 0.577 \\
$b$ & 2,718 & 1,944 & 1,720 & 1,561 & 1,437 & 1,337 & 1,248 & 1,172 & 1,104 & 1,045 & 0.992 \\
\hline
\end{tabular}

Table 3: Values of adjustable controller parameters for the desired model method

$$
\text { Control system }
$$

Controller

\begin{tabular}{|c|c|c|c|c|c|}
\hline & & & & \\
\hline & & Type & $k_{p}^{*}$ & $T_{p}^{*}$ & $T_{D}^{*}$ \\
\hline 1 & $\frac{k_{1}}{s} e^{-T_{2} s}$ & $\mathbf{P}$ & $\frac{1}{\left(\alpha T+\beta T_{d}\right) k_{1}}$ & - & - \\
\hline 2 & $\frac{k_{1}}{T_{1} s+1} e^{-T_{2} s}$ & PI (PS) & $\frac{T_{1}^{*}}{\left(\alpha T+\beta T_{d}\right) k_{1}}$ & $T_{1}-\frac{T}{2}$ & - \\
\hline 3 & $\frac{k_{1}}{s\left(T_{1} s+1\right)} e^{-T_{2} s}$ & PD & $\frac{1}{\left(\alpha T+\beta T_{d}\right) k_{1}}$ & - & $T_{1}-\frac{T}{2}$ \\
\hline 4 & $\frac{k_{1}}{\left(T_{1} s+1\right)\left(T_{2} s+1\right)} e^{-T_{2} s}$ & PID (PSD) & $\frac{T_{1}^{*}}{\left(\alpha T+\beta T_{d}\right) k_{1}}$ & $T_{1}+T_{2}-T$ & $\frac{T_{1} T_{2}}{T_{1}+T_{2}}-\frac{T}{4}$ \\
\hline & $T_{1} \geq T_{2}$ & & & & \\
\hline 5 & $k_{1}-e^{-T_{2} s}$ & SPAN (PSD) & $T_{1}^{*}$ & $2 \xi_{0} T_{2}-T$ & $\underline{T_{0}}-\underline{T}$ \\
\hline & $T_{0}^{2} s^{2}+2 \xi_{2} T_{0} s+1$ & & $\overline{\left(\alpha T+\beta T_{d}\right) k_{1}}$ & & $2 \xi_{0} \quad 4$ \\
\hline & $0.5<\xi_{0} \leq 1$ & & & & \\
\hline
\end{tabular}

Analog, $T=0$

Digital, $T>0$ 